\title{
Fatality in a Case of Envenomation by Crotalus adamanteus Initially Successfully Treated with Polyvalent Ovine Antivenom Followed by Recurrence of Defibrinogenation Syndrome
}

\author{
Craig S. Kitchens, $M D^{a, b}$, Thomas A. Eskin, $M D^{c}$
}

aDepartment of Medicine, Division of Hematology/Oncology, University of Florida College of Medicine, Gainesville, FL bMalcom Randall VA Medical Center, Gainesville, FL

cUniversity of Florida College of Medicine, Gainesville, FL

\begin{abstract}
Introduction: Recurrences of clinical or laboratory manifestations of North American pit viper envenomation may happen despite control of the envenomation syndrome by prompt and adequate antivenom therapy. Recurrences of coagulopathy in victims of Eastern diamondback rattlesnake envenomation are generally regarded as benign. The vast majority suffer no actual bleeding despite florid coagulation laboratory abnormalities due to selective defibrinogenation.

Case Report: We report what we believe to be the first fatality following successful control of the envenomation syndrome following ovine antivenom treatment resulting from envenomation by a bite from the Eastern diamondback rattlesnake. This case raises the question of whether such recurrences are in fact benign, causal, or coincidental. This patient sustained significant brain hemorrhage and death ensued due to generalized cerebral edema. Defibrinogenation occurred 4 days after treatment with ovine antivenom.

Discussion: Coagulation abnormalities following Eastern diamondback rattlesnake envenomation are due to selective defibrinogenation. This is separate from disseminated intravascular coagulation (DIC). Thrombin generation, thus hemostasis, are generally considered normal. This case may cause reexamination of this belief.
\end{abstract}

\section{INTRODUCTION}

The development of treatment of envenomation by American pit vipers has progressed from initially a nonmedical issue to a vibrant study involving clinical medicine and toxicology, producing improvements in understanding of this complex poisoning as well as improved outcome and safety [1].

Alterations in the coagulation system both in vitro and in vivo have been observed in victims of snakebite for many years [2-5]. Both hemorrhagic and thrombotic events have been described with or without accompanying laboratory perturbations. Most of these coagulation abnormalities are thought to be due to specific enzymes that vary among species that activate an array of procoagulant and/or anticoagulant aspects of hemostasis.

Local hemorrhage is characteristic of American pit viper envenomation and due in large part to local action of venom constituents that disrupt capillaries and supporting structure of the microcirculation [6,7]. In such anatomic lesions, hemostatic defects play a minimal role. Hemorrhage remote from the bite site, while reported, is unusual and apparently not a function of

Keywords: rattlesnake envenomation, defibrinogenation, hemorrhage, recurrence syndrome

Notes: This paper was not presented at any meeting or in abstract form.

There was no outside funding of any kind used for this study.

Corresponding Author: Craig S. Kitchens, MD, Malcom Randall VA Medical Center, 1601 SW Archer Road, Gainesville, FL 32608-1197. Email: craig.kitchens@medicine.ufl.edu 
alterations of the coagulation system [5]. The hemostatic defect due to Crotalus adamanteus (Eastern diamondback rattlesnake [EDR]) is one of defibrinogenation due to partial clotting of fibrinogen by the thrombin-like enzyme crotalase in that snake's venom. The laboratory abnormalities are due to plasma fibrinogen levels $<50 \mathrm{mg} / \mathrm{dl}$, resulting in very prolonged prothrombin times (PTs) and partial thromboplastin times (PTTs) without parallel alterations in coagulation factors upstream from fibrinogen within the coagulation cascade. As thrombin generation remains intact, platelet counts are not severely depressed, hemostasis remains functional, and there is no evidence for classic disseminated intravascular coagulation (DIC) [8]. The decreased fibrinogen levels are accounted for by the enormous production of fibrin (ogen) split products (FSPs) due to the brisk production of plasmin from reflex endothelial release of tissue plasminogen activator (tPA) [8].

In vitro coagulation abnormalities following antivenom therapy rapidly improve due to return of fibrinogen levels adequate (>50 mg/dL) to result in normal or near normal PTs and PTTs. However, recurrent laboratory abnormalities without clinical recrudescence of local signs and symptoms of envenomation are known to reappear after temporary correction of all coagulation abnormalities and cessation of further signs and symptoms of local pain and swelling [9]. This phenomenon is thought to be secondary to subsequent seepage of venom from deeper reservoirs in the bite site and/or disassembly of the antigen:antibody complex with reinstitution of circulating unbound venom constituents [9]. This recurrence has been described in a retrospective review following unequivocal reversal of the envenomation syndrome by the previously available equine product [10] (Antivenin [crotiladae] polyvalent, Wyeth, Marietta, PA) or particularly the recently available antivenom polyvalent crotalid (ovine) FAB (FabAV) (CroFab, Therapeutic Antibodies, Inc., Nashville, TN) [10].

\section{CASE REPORT}

A previously healthy 54-year-old man was walking in his yard on an October morning. He was bitten on the dorsum of his right hand by a positively identified 4- to 5-foot EDR. He had had a history of hepatitis $\mathrm{C}$ but no history of active liver disease, alcoholism, or substance abuse. Within 10 minutes, he requested his adult son to call for emergency medical services (EMS). EMS personnel and a photograph of the snake taken by the son helped confirm the reptile identification. The son described his father as being woozy, pale, intensely diaphoretic, and complaining of a terrible metallic taste in his mouth. The EMS records show that they arrived 20 minutes after the bite, at which time they found him "slumped back into a chair" responding very slowly to questions, and in some respiratory distress. He was pale and diaphoretic, and there was swelling and local bleeding from the back of the right hand. Once in the ground vehicle, they immediately requested air evacuation located at our medical center to meet them at a local hospital's landing zone. An IV was started and $1.5 \mathrm{~L}$ of normal saline was administered by bolus infusion. Pulse oximetry showed a room air oxygen saturation of $97 \%$. EMS personnel noticed that he was becoming hypotensive and diverted the patient directly into the smaller, local hospital for further evaluation and treatment before air evacuation. The EMS personnel also requested that 4 vials of FabAV be mixed in preparation for the patient's arrival via air. The patient then arrived at the small community medical center about 40 minutes after the bite. Blood gas analysis showed a $\mathrm{pH}$ of $7.61, \mathrm{pCO} 2$ of $18 \mathrm{mmHg}$, and pO2 of 340 $\mathrm{mmHg}$. Blood pressure was initially $109 / 66 \mathrm{mmHg}$, and during the 20-minute flight progressively dropped to $68 / 55 \mathrm{mmHg}$ despite IV fluids. He arrived at our hospital 1-1.25 hours after the accident. Facial fasciculations were noted. Infusion of the 4 vials of the premixed FabAV was immediately begun. His blood pressure promptly returned to and remained normal. He received 1-2 additional vials every 4-6 hours thereafter during the first 24 hours, for a total of $12 \mathrm{U}$ of FabAV. Coagulation studies (Table 1) of his initial pre-FabAV blood samples showed prolonged PT at 17.0 seconds and PTT of 36 seconds with a low plasma fibrinogen level of $<30 \mathrm{mg} / \mathrm{dL}$, which returned toward normal in response to the FabAV.

Table 1: Serial Coagulation Studies in Case of Recurrence Syndrome

\begin{tabular}{|c|c|c|c|c|c|c|c|c|c|c|c|c|}
\hline \multirow{3}{*}{$\begin{array}{l}\text { Day } \\
\text { Time } \\
\text { CroFab®, Vials }\end{array}$} & \multicolumn{3}{|c|}{1} & \multirow{3}{*}{$\begin{array}{c}\mathbf{2} \\
1050\end{array}$} & \multirow{3}{*}{$\begin{array}{c}3 \\
0300\end{array}$} & \multirow{3}{*}{$\begin{array}{c}4 \\
0550\end{array}$} & \multirow{3}{*}{$\begin{array}{c}5 \\
1200\end{array}$} & \multicolumn{3}{|c|}{6} & \multicolumn{2}{|c|}{7} \\
\hline & 1019 & 1417 & 2359 & & & & & 0252 & 1510 & 1952 & 0155 & $0300 \dagger$ \\
\hline & 42 & 11 & 2 & & & & & & $4^{*}$ & & & \\
\hline Platelets $\times 10^{3} / \mu \mathrm{L}$ & 109 & 195 & 231 & 208 & 163 & 143 & 126 & 106 & 179 & 120 & & \\
\hline PTT (nl, 23-33 sec) & 36 & 24 & 25 & 32 & 26 & & $>240$ & $>240$ & 25 & 22 & 23 & \\
\hline PT (nl, 10.5-13.5 sec) & 17.0 & 16.7 & 14.7 & 13.6 & 14.1 & & $>150$ & $>150$ & 16.7 & 16.7 & 17.4 & \\
\hline Fibrinogen (nl, 200-425 mg/dL) & $<30$ & 46 & 122 & 195 & 299 & & $<35$ & $<35$ & 88 & 148 & 321 & \\
\hline AT III (nl, 75-115\%) & & & & & & & 90 & & & & & \\
\hline Plasminogen (nl, 64-120\%) & & & & & & & 20 & & & & & \\
\hline d-dimer (nl, <0.48 mg/L) & 1.29 & & & & & & 0.44 & & & & & \\
\hline $\begin{array}{l}\text { *8 "units" of cryoprecipitate also adm } \\
\text { †Expired }\end{array}$ & ered & & & & & & & & & & & \\
\hline
\end{tabular}


There was no progression of the mild swelling and discoloration of the right hand over the next several days. After his initial emergency-department treatment with 4 vials FabAV there were no new or further signs of fasciculations, hemorrhage, hypotension, or progression of swelling; therefore, by all accepted criteria, his envenomation syndrome had been controlled [11]. He was followed a few days longer to observe his wound and the increase in his plasma fibrinogen levels. On Day 5 of the bite, as he prepared to go home, his PT and PTT were repeated and found to be prolonged. There were no new signs or symptoms of fasciculations, hypotension, or renewed swelling. He experienced neither headache nor mental status changes. He was not bleeding, so it was decided to follow him overnight, as is customary at this institution based on our previous lack of any hemorrhagic diatheses in this situation over approximately 100 cases of envenomation by this species. At 3:00 A.M. on the 6th day he had deterioration of his mental status, and a CT scan showed multiple small hemorrhages in the high convexity of the left and right parietal areas, as well as a separate, isolated $2 \times 2 \times 3 \mathrm{~cm}$ hemorrhage deep in the right parietal lobe. Ventricular size was normal. There was no other evidence for hemostatic failure. He was immediately administered 8 units of cryoprecipitate as a fibrinogen source and 4 more vials of FabAV, which promptly corrected his coagulopathy. His platelet count was $126,000 / \mu \mathrm{L}$ and antithrombin III level returned normal. The level of plasminogen was severely depleted, consistent with our previous reports demonstrating this event is not DIC, but selective defibrination with hyperfibrinolysis (see Table 1) [8]. That the fibrinogen level continued to increase without further cryoprecipitate administration is consistent with our prior observations that fibrinolysis was effectively controlled by the antivenom infusion. However, serial CT scans showed generalized progressive edema of the brain. Total hemorrhage did not markedly progress by radiographic monitoring at that time. A ventriculostomy was done, as well as arterial line placement without excessive hemorrhage. His mental deterioration was deduced by the neurosurgeons and neuroradiologists to be due to generalized cerebral edema and not due to solely mass effect from the primary hemorrhage. Later on the 6th day there was evidence for no cerebral blood flow, and he was declared brain dead early on the 7th day.

At autopsy, there were no obvious external signs of hemorrhage, with the exception of the right hand, the site of the snake bite. There were two unruptured hemorrhagic bullae. The arm proximal to the hand was not ecchymotic. The thoracoabdominal organs showed no evidence of hemorrhage, and the pulmonary venous return was normal. The spleen was of normal size and the liver did not show any cirrhotic changes, despite a history of prior hepatitis C. The kidneys were normal. The brain appeared grossly swollen. A ventricular shunt was still in place, and there was a small amount of subarachnoid blood present. There was bilateral uncal grooving and cerebellar tonsils were prominent, suggesting herniation. On section, the cerebral sulci and ventricles were narrowed, and there were multiple small recent intracerebral hemorrhages, including one in deep right superior parieto-occipital lobe, now measuring $5 \times 3.5 \times 2 \mathrm{~cm}$, one in inferolateral temporal white matter, now measuring $3 \times 3 \times 2 \mathrm{~cm}$, and one in left temporal lobe, now measuring $0.2 \mathrm{~cm}$ in diameter, as well as other smaller deep white matter hemorrhages. Multiple transverse sections of the brainstem and cerebellum revealed diffuse swelling of the pons and medulla. There was no evidence of trauma, tumor, cerebrovascular disease or infarction, or inflammation. The ultimate cause of death was judged to be a result of accumulated mass effect from hemorrhages and generalized edema of the brain and brainstem.

\section{DISCUSSION}

Death from American pit viper envenomation is rare and adequate autopsies even rarer. Dart and colleagues [12] reviewed the few reports regarding 16 deaths out of about 1000 cases of North American envenomations reported up to 1989. CNS edema and hemorrhage was reported in a few cases and cerebral hemorrhage was deemed to be the cause of death in one. They deduced that the most common cause was progressive shock leading to multiorgan failure and death hours to a few days later. Generalized edema from extravasation of fluid into the heart, lung, and brain were implicated. Edema is a result not of frank hemorrhage but of direct effect of the toxin on the integrity of the circulation and the microcirculation in particular.

The role of coagulopathy in the toxicology of snake envenomation is incompletely understood. Many snakes' venoms, particularly those exotic to North America, cause significant systemic hemorrhage and/or thrombosis, an unusual occurrence in envenomation by North American pit vipers [2]. White [5], in a 2005 review, correctly points out that the alterations in laboratory phenomena are considerable and often, while extremely abnormal, do not always equate with actual hemorrhagic risks, let alone events. He points out that risk stratification using prolongation of global coagulation tests cannot be inferred from extraction of one's prior experience of prolongation of these tests from excessive anticoagulant therapy, liver disease, DIC, or the hemophilias as these prolongations are due to entirely different pathologic processes, particularly those resulting in gross impairment of thrombin generation. We have previously shown that the prolongation of PTs and PTTs is inversely related solely to marked decreases in fibrinogen with preservation of other factors' activities. The inherited analogue of extremely low fibrinogen levels, congenital afibrinogenemia, is a benign disorder. It is not the prolonged PT and PTT that result in hemorrhagic or thrombotic potential, but the cause of those prolongations [13].

Recurrence of clinical and laboratory manifestations following what is usually regarded as adequate antivenom therapy is of concern. The clinical significance of the recurrence, particularly as manifest by return of coagulation abnormalities from North American pit vipers, is not at all understood. These recurrences have been best defined as the result of follow-up of patients bitten by North American pit vipers, particularly rattlesnakes, since the advent of FabAV. This discovery was enabled because of the 
research, development, and observation in clinical protocoldriven trials of patients treated with FabAV, which garnered the largest and most extensively followed group of such patients [9]. In retrospective studies, Bogdan and others [10] found that there were data that among 354 consecutive patients treated for North American crotalid bites, 112 exhibited coagulopathy. Of these, 31 had undergone coagulation testing sufficient to detect whether a recurrence occurred; of the 31, 14 (45\%) had a recurrence of the coagulopathy to include severe hypofibrinogenemia or thrombocytopenia, yet none of these patients experienced spontaneous hemorrhage despite these laboratory recurrences.

Boyer and colleagues [9], in studying FabAV-treated crotalid envenomations in 38 patients, found that 20 (53\%) had recurrent, persistent, or late coagulopathy, some occurring up to 13 days following envenomation and treatment. No patient experienced significant spontaneous bleeding. The most common severe abnormality was incoagulable and/or extremely prolonged PTs or PTTs, all of which were due to severe selective defibrinogenemia. Of their 20 patients, 16 were observed with no further treatment, and all fared well. Two patients who received supplemental doses of FabAV experienced normalization of laboratory findings, much as this reported patient did. Of interest, all their patients with defibrinogenemia on presentation showed significant increases in their plasma fibrinogen levels following FabAV treatment, which is the major laboratory criterion for a therapeutic response to FabAV. They noted that the local bite injury, which was controlled by the initial treatment of FabAV, did not advance despite the late recurrence of coagulopathy.

Regarding our case, there seem to be several possible explanations. First, it is possible that despite receiving adequate doses of antivenom within 80 minutes of the accident, during the initial phase of his severe bite he developed multiple CNS hemorrhages without our detection, which then may have re-bled five days later. A second possibility is that during the height of his severe envenomation syndrome independent of coagulation laboratory changes, microvascular damage sufficient to cause capillary leak resulting in gross cerebral edema, increased pressure, decreased perfusion, and eventual brain death. Third, the event could have been due solely to the return of the coagulopathy which caused his death. A fourth possibility is that the patient had suffered some unknown preexisting CNS lesion (e.g., trauma, tumor, stroke, or infection) that then served as a nidus for subsequent hemorrhage yet lacked any evidence at autopsy. As any of the first three mechanisms seems plausible in this case, it is clear that we cannot be sure which of these (along with the fourth possibility) represents the chief risk, let alone how to minimize or treat that risk.
It is unclear what courses of action to recommend. It is clear that late recurrences, especially involving late defibrinogenation, may occur after unambiguous control of the envenomation syndrome achieved by the initial aggressive antivenom treatment. Whether defibrinogenation itself causes remote delayed hemorrhage is uncertain but, if so, it seems to be exceedingly rare.

\section{REFERENCES}

1. Kitchens CS. From EtOH to Fab: the medicalization of therapy for pit viper envenomation. Trans Am Clin Climatol Assoc 2001;112:117-135.

2. Kitchens CS. Hemostatic aspects of envenomation by North American snakes. Hematol/Oncol Clin NA 1992;6: 1189-1195.

3. Van Mierop LHS. Poisonous snakebite: a review, 1: snakes and their venom. J Fla Med Assoc 1976;63:191-199.

4. Budzynski AZ, Pandya BV, Rubin RN, Brizuela BS, Soszka T, Stewart GJ. Fibrinogenolytic afibrinogenemia after envenomation by Western diamondback rattlesnake (Crotalus atrox). Blood 1984;63:1-14.

5 . White J. Snake venoms and coagulopathy. Toxicon 2005;45:951-967.

6. Gold BS, Dart RC, Barish RA. Bites of venomous snakes. N Engl J Med 2002;347:347-356.

7. Ownby C. Pathology of rattlesnake envenomation. In: Tu AT, ed. Rattlesnake Venoms. New York: Marcel Dekker, 1982;163-209.

8. Kitchens CS, Van Mierop LHS. Mechanism of defibrination in humans after envenomation by the Eastern diamondback rattlesnake. Am J Hematol 1983;14:345-353.

9. Boyer LV, Seifert SA, Clark RF, McNally JT, Williams SR, Nordt SP, et al. Recurrent and persistent coagulopathy following pit viper envenomation. Arch Intern Med 1999;159:706-710.

10. Bogdan GM, Dart RC, Falbo SC, McNally J, Spaite, D. Recurrent coagulopathy after antivenom treatment of crotalid snakebite. South Med J 2000;93:562-566.

11. Dart RC, Hurlbut KM, Garcia RA, Boren JL. Validation of a severity score for the assessment of crotalid snakebite. Ann Emerg Med 1996;27:321-326.

12. Dart RC, McNally JT, Spaite DW, Gustafson R. The sequelae of pitviper poisoning in the United States. In: Campbell JA, Brodie, ED Jr, eds. Biology of Pit Vipers. Tyler TX, Selva Publications, 1992;395-404.

13. Kitchens CS. To bleed or not to bleed? Is that the question for the PTT? J Thromb Haemost 2005;3:2607-2611. 